Method Discrete compartmental models are defined by a set of ordered states (compartments) reflecting the health status, and can be fully characterised by the set of transition probabilities between each compartment. When defined at the individual level, each participant contributes to the likelihood of the model at each year from the time of entering the initial stage (e.g. birth) to the moment they reach an absorbing state (e.g. death or clinical onset). Model estimation aims at quantifying the transitions ensuring the best reconstruction of the pathological trajectories in each subject, hence adding to the classification problem (discriminating healthy and diseased subjects) a dynamic component (estimating the time of onset).

Individual exposure histories can be summarised through cumulative exposure functions and subsequently plugged into the compartmental framework as parameters of transition probabilities.

Results While these models were initially developed to accommodate data from longitudinal studies, we will illustrate, using lung cancer case control and smoking history data, the validity and utility of such approaches. We will assess the underlying assumptions yielded by this methodological drift and will exemplify the rich statistical inference these approaches are able to provide.

Conclusions We will finally introduce potential extensions over this framework that include omics biomarkers to model genetically-driven susceptibility and/or to identify the stage (s) at which exposure (s) are more likely to mediate their effects.

\section{LONG NIGHT SHIFTS AMONG HEALTH WORKERS AND PHYSICAL AND MENTAL HEALTH: THE INFLUENCE OF ON-SHIFT NAP AND DOMESTIC WORK}

Lucia Rotenberg, Rosane Griep, Aline Silva-Costa, Luciana Portela, Thiago Diniz, Adenilda Arruda. Oswaldo Cruz Foundation, Rio de Janeiro, Brazil

\subsection{6/oemed-2014-102362.388}

Objectives This presentation during the Shift work Symposium aims to discuss data on physical and mental health among health workers, considering the relevance of on-shift naps and routine housework. In addition, the presentation aims also to analyse the cumulative exposure to night work.

Method Data to be presented are based on several epidemiological studies among nursing teams working at 18 Brazilian public hospitals. Databank includes information on socio-demographic and health. Data on work refers to the allowance (or not) to take naps during the night shift and nap regularity. Occupational history data considers (i) whether day workers have worked at night in the past and for how long and (ii) the reasons for quitting night work among former night workers.

Results The allowance for taking naps was observed in all studied hospitals. On-shift nap is a frequent practice among nursing workers. The analysis of occupational history revealed to be relevant as regards physical and mental health. Among former night workers, those who quitted night work for health reasons are at a higher risk of reporting mental suffering.

Conclusions Housework demands seem to aggravate sleep deprivation related to night work, despite evidences of beneficial effects of on-shift naps on workers' recovery. The specific study of former night workers has revealed to be a fruitful approach in studies on health, obesity included, lifestyle and habits, as well as sleep disturbances. Occupational history is an adequate approach for a comprehensive understanding of the impact of night work on health.

\section{CORONARY ARTERY DISEASE MORTALITY AMONG WORKERS EXPOSED TO CARBON DISULFIDE AND SHIFT WORK AT A CHEMICAL MANUFACTURING PLANT}

${ }^{1}$ Tania Carreón, ${ }^{1}$ Misty Hein, ${ }^{1}$ Kevin Hanley, ${ }^{2}$ Susan Viet, ${ }^{1}$ Avima Ruder. ${ }^{1}$ National Institute for Occupational Safety and Health, Cincinnati, Ohio, USA; ${ }^{2}$ Westat, Bethesda, Maryland, USA

\subsection{6/oemed-2014-102362.389}

Objectives Previous studies at a New York State chemical manufacturing plant reported elevated risks of cardiovascular disease among workers. We updated the mortality experience of 1874 workers employed between 1949 and 2006 through December 31, 2007. We investigated exposures to carbon disulfide and shift work and their association with coronary artery disease.

Method Jobs with carbon disulfide and shift work exposure $(\geq 1$ day) were identified among departments and job titles in specific years. Standardised mortality ratios (SMR) compared mortality to the US population, adjusted for gender, race, age, and calendar year. Internal comparisons used directly standardised rate ratios (SRR).

Results Overall, excess deaths were observed for coroanary artery disease $(\mathrm{SMR}=1.24,95 \%$ CI 1.04-1.48). Most workers exposed to carbon disulfide perfortmed shift work; we evaluated coronary artery disease mortality in groups defined by duration of exposure to these agents. Compared to the US population, statistically significant increases in mortality were observed among workers with both exposures for 90 days or more ( $\mathrm{SMR}=1.36,95 \%$ CI 1.03-1.76), and among workers with fewer than 90 days of both exposures (SMR $=1.31,95 \% \mathrm{CI}$ 0.65-2.34). Using cutpoints of 4 years (median exposure duration among long-term cases), the results were no longer statistically significant. In internal comparisons, long-term workers exposed to carbon disulfide and shift work for 4 years or more had a near 3 -fold increase in coronary artery disease mortality, compared to workers exposed less than 4 years.

Conclusions Excess coronary artery disease mortality confirms earlier results, but further investigation is needed to understand risk factors.

\section{INDIVIDUAL VARIABILITY, FROM CANDIDATE G*E TO GEWIS}

Manolis Kogevinas. Centre for Research in Environmental Epidemiology (CREAL, Barcelona, Spain)

10.1136/oemed-2014-102362.390

Objectives In the 1990s there were great expectations that the use of markers of genetic susceptibility would allow the identification of new occupational risks, a more complete characterisation of dose-response relationships and improved risk assessment. Several interaction studies were conducted examining candidate genes, for example on isocyanate exposure, genes in immune pathways (e.g. HLAII group) and occupational asthma, or studies on cancer, aromatic amines and the NAT2 gene.

Method Very few replicated in more than one population. Part of the problem was the small sample size and the selective 